\title{
Clinical Characteristics and Efficacy of Iron Treatment for Restless Legs Syndrome Patients with Very Low Ferritin Levels
}

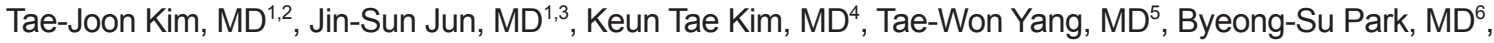 \\ Jung-Ah Lim, $M D^{7}$, Jung-Ick Byun, $M^{8}{ }^{8}$, Jun-Sang Sunwoo, $M^{9}{ }^{9}$, Jung-Won Shin, $\mathrm{MD}^{10}$, Ki-Young Jung, MD, $\mathrm{PhD}^{1}$ \\ 'Department of Neurology, Seoul National University Hospital, Seoul, Korea \\ ${ }^{2}$ Department of Neurology, National Center for Mental Health, Seoul, Korea \\ ${ }^{3}$ Department of Neurology, School of Medicine, Kyungpook National University, Kyungpook National University Chilgok Hospital, Daegu, Korea \\ ${ }^{4}$ Department of Neurology, Keimyung University Dongsan Medical Center, Daegu, Korea \\ ${ }^{5}$ Department of Neurology, Gyeongsang National University Changwon Hospital, Gyeongsang National University School of Medicine, Changwon, Korea \\ ${ }^{6}$ Department of Neurology, Ulsan University Hospital, Ulsan, Korea \\ ${ }^{7}$ Department of Neurology, Kangnam Sacred Heart Hospital, Hallym University College of Medicine, Seoul, Korea \\ ${ }^{8}$ Department of Neurology, Kyung Hee University Hospital at Gangdong, Seoul, Korea \\ ${ }^{9}$ Department of Neurology, Soonchunhyang University School of Medicine, Seoul, Korea \\ ${ }^{10}$ Department of Neurology, CHA Bundang Medical Center, CHA University, Seongnam, Korea
}

Received: May 21, 2018

Revised: July 13, 2018

Accepted: November 26, 2018

Correspondence

Ki-Young Jung, $\mathrm{MD}, \mathrm{PhD}$

Department of Neurology,

Seoul National University Hospital,

Seoul National University

College of Medicine,

101 Daehak-ro, Jongno-gu,

Seoul 03080, Korea

Tel $\quad+82-2-2072-4988$

Fax +82-2-2072-7424

E-mail jungky@snu.ac.kr

ORCID

Tae-Joon Kim

https://orcid.org/0000-0001-8451-6634 Jin-Sun Jun

https://orcid.org/0000-0001-9879-0634 Keun Tae Kim

https://orcid.org/0000-0002-7124-0736

Tae-Won Yang

https://orcid.org/0000-0002-8113-2384

Byeong-Su Park

https://orcid.org/0000-0003-0397-7725

Jung-Ah Lim

https://orcid.org/0000-0001-5292-385X Jung-Ick Byun

https://orcid.org/0000-0002-6224-4575

Jun-Sang Sunwoo

https://orcid.org/0000-0002-8168-3127

Jung-Won Shin

https://orcid.org/0000-0003-2155-9068

Ki-Young Jung

https://orcid.org/0000-0001-5528-9081
Restless legs syndrome (RLS) is associated with dopaminergic dysfunction, and iron deficiency, manifesting as low ferritin. However, ferritin levels do not correlate well with demographic data. Based on the hypothesis that very low ferritin can accurately reflect clinical information, we herein described characteristics and treatment efficacy of RLS patients with ferritin levels under $15 \mathrm{ng} / \mathrm{mL}$. We retrospectively reviewed 196 patients and collected clinical data. Twenty-one patients had ferritin levels under $15 \mathrm{ng} / \mathrm{mL}$ and were compared with those having ferritin levels over $15 \mathrm{ng} / \mathrm{mL}$. The patients with very low ferritin levels were predominantly female, had earlier onset of symptoms, and showed a superior response to iron therapy. In conclusion, RLS patients with ferritin levels under $15 \mathrm{ng} / \mathrm{mL}$ showed distinct demographic features and treatment efficacy. Further studies on more patients with long-term follow-up are warranted to support this observation.

Sleep Med Res 2018;9(2):118-123

Key Words Restless legs syndrome, Ferritin, Iron.

\section{INTRODUCTION}

Restless legs syndrome (RLS) is a neurological sensorimotor disorder characterized by an urge to move the legs in a circadian pattern, usually accompanied by uncomfortable sensation [1]. Treatment is essential since RLS patients often also have insomnia, depression, and low quality of life [2]. The symptoms of RLS patients are known to derive from dopaminergic dysfunction, and brain iron deficiency manifesting as a low ferritin level, and treatments for RLS patients also target such pathomechanisms [3]. Ferritin is a key factor in iron homeostasis and may be related to the clinical manifestations of RLS. Ferritin levels have a modest inverse correlation with the severity of patients' symptoms [4,5]. Recently, a few studies revealed that iron therapy was effective for RLS patients [6,7]. However, previous studies have failed to show that low ferritin levels (less than $45-50 \mathrm{ng} / \mathrm{mL}$ ) were associated with the demographic characteristics.

Ferritin levels have not been associated with various clinical features, possibly due to study methodology as well as iron availability to the brain. Ferritin, an iron storage protein in the pe- 
ripheral body and the central nervous system, is a useful indicator of iron deficiency. A systemic iron deficiency with low serum ferritin leads to decreased brain iron levels; however, the brain-specific iron deficit can also originate from the regulation of iron transport through the blood-brain barrier [8]. Cerebrospinal fluid (CSF) levels of ferritin have been shown to be decreased in RLS patients compared with controls, even when no difference in serum ferritin levels existed [9]. Furthermore, a brain autopsy study revealed a defect in the regulation of iron transport receptors in RLS, indicating impaired iron acquisition in the brain $[10,11]$. Therefore, serum ferritin levels and clinical characteristics may not be directly correlated and can have complex relationships. In our clinical experience, the RLS patients with very low ferritin levels showed good responses to iron therapy, most of whom were young women, and these two were unique characteristics of the group.

Therefore, we hypothesized that ferritin levels could clearly distinguish groups of RLS patients, if the appropriate ferritin cut-off level was established. We set a cut-off ferritin level of 15 $\mathrm{ng} / \mathrm{mL}$, a value generally used for diagnosis of iron deficiency anemia [12], for the purpose of comparing patients with very low ferritin levels to those who had not. Accordingly, we investigated the clinical characteristics and iron treatment efficacy of RLS patients with very low ferritin levels under $15 \mathrm{ng} / \mathrm{mL}$.

\section{METHODS}

This study retrospectively reviewed the medical records of patients with RLS who visited the outpatient clinic of the Department of Neurology at Seoul National University Hospital from March 2014 to April 2018. RLS was diagnosed according to the 2003 diagnostic criteria of the International RLS Study Group [13]. Clinical data of RLS patients with serum ferritin levels under $15 \mathrm{ng} / \mathrm{mL}$ (very low ferritin, VLF group) were compared with those having ferritin levels $\geq 15 \mathrm{ng} / \mathrm{mL}$ (non-VLF group). Clinical information gathered on RLS patients consisted of demographic features, clinical presentations, laboratory tests, sleep questionnaires, and past medical history. Demographic information included age, sex, and body mass index (BMI). Clinical presentations associated with RLS were also collected. Laboratory tests included serum ferritin and hemoglobin levels. Anemia was defined as a hemoglobin level $<12 \mathrm{~g} / \mathrm{dL}$ in women and $<13 \mathrm{~g} / \mathrm{dL}$ in men. Sleep questionnaires included the Pittsburgh Sleep Quality Index (PSQI), Epworth Sleepiness Scale (ESS), Insomnia Severity Index (ISI), Beck Depression Inventory (BDI), International RLS study group rating scale, and RLS Quality of Life scale. Poor sleep quality was defined as a PSQI score $>5$. Daytime sleepiness was measured with the ESS, and excessive daytime sleepiness was defined as a score $>10$. Presence of insomnia was indicated by an ISI score higher than 14 . Depressive symptoms were evaluated with the BDI, with a cut- off score of 10. Past medical history collected on patients included diabetes, hypertension, and chronic kidney disease.

For evaluating treatment efficacy of iron replacement therapy, the VLF group was compared with the low ferritin group (LF group, ferritin level between 15 and $75 \mathrm{ng} / \mathrm{mL}$ ), since the recent guideline suggests intravenous (IV) iron therapy in RLS patients with ferritin $\leq 75 \mathrm{ng} / \mathrm{mL}$ [14]. If a patient underwent IV iron therapy, the injection protocol of $1000 \mathrm{mg}$ infusion of ferric carboxymaltose mixed with normal saline over 30 minutes was used. The response of treatment was evaluated with the clinical global impression (CGI) rating scale at the next outpatient clinic visit. The CGI scale ranges from 1 point, indicating very much improved, to 7 points, indicating very much worse. The exact time-point of CGI evaluation was retrospectively checked. Concomitant prescriptions for RLS symptoms were also collected during IV iron infusion. This retrospective study was approved by the Institutional Review Board of Seoul National University Hospital (IRB no. 1806-168-954), and the requirement to obtain informed consent was waived.

Statistical comparison between the two groups was performed using nonparametric methods. For categorical data, a contingency table was created, and Fisher's exact test was performed. Bonferroni's correction was applied for multiple comparisons in the number of iron treatments and treatment efficacy according to the CGI scale. In addition, ordinal logistic regression was employed to examine the independent predictors of treatment efficacy. Multivariable models included treatment efficacy (CGI scale) as a dependent variable and each group (VLF vs. LF group) as an independent variable, along with age, sex, and other comorbidities as covariates. The Mann-Whitney U-test was used for comparison of continuous variables or discrete variables, such as questionnaire scores. Data were expressed as a percentage of the available subjects or mean \pm standard deviation. All statistical analyses were performed using IBM SPSS Statistics version 23.0 (IBM Co., Armonk, NY, USA), and a two-tailed p-value of less than 0.05 was considered statistically significant.

\section{RESULTS}

A total of 196 patients with RLS were enrolled in the study, 21 (11\%) of whom had ferritin levels under $15 \mathrm{ng} / \mathrm{mL}$ and belonged to the VLF group (Table 1). The patients in the VLF group were significantly younger, compared to those in the non-VLF group (age $48.4 \pm 16.3$ yrs vs. $61.5 \pm 13.8$ yrs; $\mathrm{p}<0.001$ ). Most of the VLF group were women; therefore, the VLF group had a significantly higher percentage of women than the non-VLF group ( 20 of $21,95 \%$ vs. 102 of $175,58 \% ; p=0.001$ ). The hemoglobin levels of the VLF group were significantly lower than those of the non-VLF group $(11.8 \pm 1.7 \mathrm{~g} / \mathrm{dL}$ vs. $13.7 \pm 1.4 \mathrm{~g} / \mathrm{dL} ; \mathrm{p}<0.001)$, and there were more anemic patients in the VLF group. RLS symptoms in the VLF group occurred at younger ages (36.8 \pm 
Table 1. Clinical characteristics of RLS patients with very low ferritin levels $(<15 \mathrm{ng} / \mathrm{mL})$

\begin{tabular}{|c|c|c|c|}
\hline & Ferritin $<15 \mathrm{ng} / \mathrm{mL}$ group $(\mathrm{n}=21)$ & Ferritin $\geq 15 \mathrm{ng} / \mathrm{mL}$ group $(\mathrm{n}=175)$ & $\mathrm{p}$ \\
\hline \multicolumn{4}{|l|}{ Demographic factors } \\
\hline Age (yrs) & $48.4 \pm 16.3$ & $61.5 \pm 13.8$ & $<0.001^{*}$ \\
\hline Sex (female, \%) & $20 / 21(95)$ & $102 / 175(58)$ & $0.001^{*}$ \\
\hline BMI $\left(\mathrm{kg} / \mathrm{m}^{2}\right)$ & $22.1 \pm 3.1$ & $23.2 \pm 2.9$ & 0.140 \\
\hline \multicolumn{4}{|l|}{ Lab tests } \\
\hline Ferritin (ng/mL) & $8.5 \pm 4.2$ & $97.4 \pm 88.5$ & $<0.001^{*}$ \\
\hline Hemoglobin $(\mathrm{g} / \mathrm{dL})$ & $11.8 \pm 1.7$ & $13.7 \pm 1.4$ & $<0.001^{*}$ \\
\hline Anemia $^{\dagger}$ & $9 / 21(43)$ & $21 / 175(12)$ & $0.001^{*}$ \\
\hline \multicolumn{4}{|l|}{ RLS characteristics } \\
\hline Onset age (yrs) & $36.8 \pm 18.9$ & $47.7 \pm 17.3$ & $0.008^{*}$ \\
\hline Early-onset $^{\dagger}$ & $15 / 20(75)$ & $56 / 160(35)$ & $0.001^{*}$ \\
\hline Symptom durations (yrs) & $11.7 \pm 13.4$ & $14.0 \pm 12.5$ & 0.205 \\
\hline Family history & $9 / 21(43)$ & $51 / 175(29)$ & 0.215 \\
\hline Aggravation during menstruation & $3 / 20(15)$ & $8 / 102(8)$ & 0.386 \\
\hline Unilateral Sx & $2 / 21(10)$ & $15 / 175(9)$ & 1.000 \\
\hline Augmentation & $2 / 21(10)$ & $27 / 175(16)$ & 0.745 \\
\hline Follow-up (months) & $12.2 \pm 16.1$ & $13.5 \pm 14.5$ & 0.705 \\
\hline \multicolumn{4}{|l|}{ Questionnaires (drug-naïve) $)^{\ddagger}$} \\
\hline Poor sleep quality & $11 / 14(79)$ & $148 / 155(93)$ & $0.039^{*}$ \\
\hline Excessive daytime sleepiness & $5 / 15(33)$ & $35 / 158(22)$ & 0.342 \\
\hline Insomnia & $9 / 17(53)$ & $101 / 165(61)$ & 0.604 \\
\hline Depression & $11 / 14(79)$ & $108 / 158(68)$ & 0.554 \\
\hline IRLS score & $26.5 \pm 8.4$ & $27.7 \pm 8.0$ & 0.321 \\
\hline RLS-QoL score & $58.1 \pm 18.2$ & $58.0 \pm 22.8$ & 0.978 \\
\hline \multicolumn{4}{|l|}{ Medical history } \\
\hline Diabetes & 0 & $18 / 175(10)$ & 0.227 \\
\hline Hypertension & $2 / 21(10)$ & $43 / 175(25)$ & 0.170 \\
\hline $\mathrm{CKD}$ & 0 & $4 / 175(2)$ & 1.000 \\
\hline
\end{tabular}

Data are presented as mean \pm standard deviation or number (percentage).

${ }^{*} \mathrm{p}<0.05 .{ }^{\dagger}$ Anemia was defined as a hemoglobin level $<12 \mathrm{~g} / \mathrm{dL}$ in females and $<13 \mathrm{~g} / \mathrm{dL}$ in males. Early-onset was defined as an onset age is less than 45 years old. $¥$ Definition: Poor sleep quality, Pittsburgh Sleep Quality Index $>$ 5; Excessive daytime sleepiness, Epworth Sleepiness Scale > 10; Insomnia, Insomnia Severity Index > 14; Depression, Beck Depression Inventory $\geq 10$.

BMI: body mass index, RLS: restless legs syndrome, IRLS: International RLS study group rating scale, RLS-QoL: RLS Quality of Life scale, CKD: chronic kidney disease.

18.9 yrs vs. $47.7 \pm 17.3$ yrs; $\mathrm{p}=0.008)$. RLS symptoms were aggravated during menstruation more in the VLF group but did not reach statistical significance. BMI, RLS symptom duration, family history, and augmentation were not significantly different between the two groups. The VLF group had no special medical history, except for hypertension in two patients.

If the ferritin cut-off level was conventionally set to $45 \mathrm{ng} /$ $\mathrm{mL}, 68$ of 196 patients whose ferritin levels were lower than 45 $\mathrm{ng} / \mathrm{mL}$ were younger $(56.5 \pm 15.2$ yrs vs. $62.0 \pm 14.0 \mathrm{yrs} ; \mathrm{p}=$ $0.009)$ and predominantly female $(76 \%$ vs. $55 \%, \mathrm{p}=0.003)$, compared with patients having ferritin $\geq 45 \mathrm{ng} / \mathrm{mL}$, but the differences between the groups were decreased. Moreover, the symptom onset was not significantly earlier (44.4 \pm 17.7 yrs vs. $47.6 \pm 17.8$ yrs, $\mathrm{p}=0.189$ ) and other conditions did not differ, although aggravation of RLS symptoms during menstruation became significant at lower levels of ferritin ( $17 \%$ vs. $3 \%, \mathrm{p}=$ 0.009).

The VLF group complained of poor sleep quality less than the non-VLF group (79\% vs. 93\%, p = 0.039), as assessed by the PSQI score. Questionnaire results regarding excessive daytime sleepiness, insomnia, and depression did not show significant differences. There was no significant difference in the RLS severity or quality of life due to RLS symptoms between the two groups. 
Interestingly, the efficacy of iron treatment for the VLF group was superior to that for the LF group with ferritin levels between 15 and $75 \mathrm{ng} / \mathrm{mL}$ (Table 2). Fourteen of 21 (67\%) patients in the VLF group and 51 of 100 (51\%) in the LF group received IV iron therapy. Among the 65 patients who received IV iron, 48 (74\%) received a single injection of ferric carboxymaltose 1000 $\mathrm{mg}$, and the remaining 17 (26\%) patients received 2-5 courses of injections. There was no significant difference in the doses between the two groups. Neither concomitant medications nor the time point of CGI evaluation differed between VLF and LF groups. Of the 14 patients who received IV iron therapy and were clinically evaluated in the VLF group, five were very much improved (CGI 1), three much improved (CGI 2), and four minimally improved (CGI 3). In contrast, most treated patients of the LF group had CGI scores between 2 and 4 . As a result, the VLF group had a significantly better effect from IV iron treatment than the LF group ( $\mathrm{p}=0.001)$. After performing ordinal logistic regression analysis, the treatment efficacy of iron therapy was significantly associated with only one variable, that is, groups (beta $=0.44,95 \%$ confidence interval 0.35-2.12, $\mathrm{p}=0.008$ ). Covariates such as age, sex, and other comorbidities did not affect the treatment results.

\section{DISCUSSION}

The present study classified RLS patients in relation to a ferritin cut-off level of $15 \mathrm{ng} / \mathrm{mL}$, and demonstrated that patients with very low ferritin levels had significantly different demographics and efficacy of iron therapy, compared to those with ferritin levels over $15 \mathrm{ng} / \mathrm{mL}$. Specifically, RLS patients with very low ferritin levels were younger and predominantly female, and symptoms began at a younger age. Moreover, IV iron treatment was more effective in patients with very low ferritin levels. These results indicate that the grouping of RLS patients by a ferritin cut-off value of $15 \mathrm{ng} / \mathrm{mL}$ was clinically meaningful.

In previous studies, the demographic characteristics of RLS patients with different ferritin levels were not apparently evaluated. One study of 18 elderly patients showed that serum ferritin levels were inversely correlated with the severity of RLS symptoms, and iron therapy was effective in patients with ferritin levels lower than a $45 \mathrm{ng} / \mathrm{mL}$ cut-off [4]. Another study set the ferritin cut-off level at $50 \mathrm{ng} / \mathrm{mL}$, and showed that patients with low ferritin levels showed greater RLS severity, lower sleep efficiency, and more periodic leg movements with arousal [5]. Another cohort study found that a periodic limb movement index $\geq 15$ was associated with low ferritin levels ( $\leq 50 \mathrm{ng} / \mathrm{mL}$ ), after controlling for cofactors [15]. However, these studies did not consider different demographic characteristics of RLS patients

Table 2. Iron treatment efficacy of restless legs syndrome patients with very low ferritin $(<15 \mathrm{ng} / \mathrm{mL})$ versus those with low ferritin $(15-75$ $\mathrm{ng} / \mathrm{mL}$ )

\begin{tabular}{|c|c|c|c|}
\hline & Ferritin $<15$ ng/mL group $(\mathrm{n}=21)$ & Ferritin $15-75$ ng/mL group $(\mathrm{n}=100)$ & $\mathrm{p}$ \\
\hline \multicolumn{4}{|l|}{ Clinical characteristics } \\
\hline Age (yrs) & $48.4 \pm 16.3$ & $61.8 \pm 14.0$ & $<0.001^{*}$ \\
\hline Sex (female, \%) & $20 / 21(95)$ & $69 / 100(69)$ & $0.008^{*}$ \\
\hline IRLS score & $26.5 \pm 8.4$ & $28.5 \pm 7.1$ & 0.426 \\
\hline Number of patients treated with IV iron & $14 / 21(67)$ & $51 / 100(51)$ & 0.233 \\
\hline Number of IV iron replacement & $1.9 \pm 1.3$ & $1.4 \pm 0.7$ & 0.066 \\
\hline \multicolumn{4}{|l|}{ Concomitant medications } \\
\hline Dopamine agonist & $9 / 14(64)$ & $36 / 51(71)$ & 0.747 \\
\hline Alpha-2-delta ligand & $5 / 14(36)$ & $30 / 51(59)$ & 0.143 \\
\hline Benzodiazepine & $1 / 14(7)$ & $10 / 51(20)$ & 0.432 \\
\hline Opioid & 0 & $4 / 51(8)$ & 0.569 \\
\hline Time point of CGI evaluation (months) & $1.3 \pm 0.5$ & $1.9 \pm 1.3$ & 0.279 \\
\hline Iron treatment efficacy & & & $0.001^{*}$ \\
\hline CGI 1, very much improved & $5 / 12(42)$ & $1 / 45(2)$ & \\
\hline CGI 2, much improved & $3 / 12(25)$ & $18 / 45(40)$ & \\
\hline CGI 3, minimally improved & 4/12 (33) & $11 / 45(24)$ & \\
\hline CGI 4 , no change & 0 & $14 / 45(31)$ & \\
\hline CGI 5, minimally worse & 0 & $1 / 45(2)$ & \\
\hline
\end{tabular}

Data are presented as mean \pm standard deviation or number (percentage).

${ }^{*} \mathrm{p}<0.05$.

IRLS: International RLS study group rating scale, IV: intravenous, CGI: clinical global impression. 
with low ferritin levels, since the studies set the cut-off level at 45-50 ng/mL, and the first study was conducted only on elderly patients. The present study collected data on all the adult RLS patients at one tertiary center and divided the patients into two groups according to a ferritin cut-off level of $15 \mathrm{ng} / \mathrm{mL}$, then observed distinct features in the group with very low ferritin levels. When the ferritin cut-off level was set to $45 \mathrm{ng} / \mathrm{mL}$ in our study, the clear differences seen between groups when the cut-off was set to $15 \mathrm{ng} / \mathrm{mL}$ were alleviated or disappeared.

In this study, the RLS patients with very low ferritin levels ( $<15$ $\mathrm{ng} / \mathrm{mL}$ ) had early-onset symptoms, female predominance, and less poor sleep quality. Using CSF or magnetic resonance image of RLS patients, Earley et al. [16,17] found that the earlyonset RLS group had significantly lower CSF ferritin levels and lower iron index in the substantia nigra, consistent with our observations. They also showed that women had significantly lower ferritin levels than men [16]. Most epidemiologic studies have reported an RLS prevalence approximately two times higher in women than in men [2], in accordance with the effects of menstrual iron loss during reproductive years [18]. The proportion of RLS patients reporting poor sleep quality was lower in the VLF group, although the absolute number of poor-quality sleepers was already high (79\%). In the literature, more than $60 \%$ of RLS subjects complained of poor sleep quality, which was more frequent in patients with severe RLS symptoms [19]. In this study, the non-VLF group was older and had symptoms of longer duration, which may affect sleep quality results. In addition, $20-30 \%$ of the patients had excessive daytime sleepiness, approximately half of them had insomnia, and $70-80 \%$ reported a depressive mood, although these results did not differ significantly between the two groups.

RLS patients with very low ferritin levels had superior responses to iron replacement therapy. Two recent controlled studies showed the efficacy of IV iron therapy for treating RLS patients; however, these studies recruited RLS patients with a wide range of serum ferritin levels from $5 \mathrm{ng} / \mathrm{mL}$ to $153 \mathrm{ng} / \mathrm{mL}$, and there was no relation between outcomes and pretreatment serum ferritin levels in either study $[6,7,20]$. From this evidence, the most recent guidelines for the iron treatment of RLS could not definitively set the ferritin level of a good responder [20]. Although the present study was retrospective and included anemic patients, its results can provide a good predictor of IV iron therapy efficacy in RLS patients. In the future, molecular, genetic, and pathologic studies of RLS patients with low ferritin levels are needed to correlate with the blunted serum-to-CSF ferritin relationship that suggests an alteration in the blood-brain barrier iron transport mechanism in RLS patients may affect treatment efficacy $[9,16]$.

This study has several limitations. First, the number of patients in the VLF group was relatively small. Second, this study did not observe the difference in augmentation rate, although previous studies have reported that augmentation in RLS was associated with low ferritin levels [21]. This may be due to the short follow-up period of approximately twelve months. Third, limitations may be derived from the retrospective study design. For example, the time point of the CGI evaluation was different between individuals due to interval variation between outpatient clinic visits, and retrospective study design. Finally, it can be said that the VLF group in this study only reflected the characteristics of younger females with a sleep disorder, considering the high prevalence of anemia or low ferritin in females of reproductive age [12]. However, approximately half of the patients in the VLF group were not anemic and otherwise healthy. Moreover, not all the young females with anemia or low ferritin had RLS symptoms. In short, this study aimed to emphasize the demographic characteristics of the VLF group of RLS patients in a tertiary hospital.

In conclusion, we showed that RLS patients with ferritin levels under $15 \mathrm{ng} / \mathrm{mL}$ were characterized by early-onset symptoms, female predominance, and an excellent response to iron therapy. These results can provide implications for clinical evaluations and help to make therapeutic decisions for RLS patients. Further prospective studies with a large cohort of RLS patients are warranted to support these findings.

\section{Acknowledgments}

This work was supported by the National Research Foundation of Korea (NRF) grants funded by the Minister of Science, ICT and Future Planning, Republic of Korea (No. NRF-2017R1A2B2012280; NRF-2017R1D1A1B030 29293; NRF-2017M3C7A1029485).

\section{Conflicts of Interest}

The authors have no financial conflicts of interest.

\section{Authors' Contribution}

Conceptualization: Jung KY. Data curation: Kim TJ, Jun JS, Kim KT, Yang TW, Park BS, Lim JA, Byun JI, Sunwoo JS, Shin JW. Formal analysis: Kim TJ. Investigation: Kim TJ, Jun JS, Kim KT, Yang TW, Park BS, Lim JA, Byun JI, Sunwoo JS, Shin JW. Writing_original draft: Kim TJ. Writing—review \& editing: Kim TJ, Jung KY. Supervision: Jung KY.

\section{REFERENCES}

1. Allen RP, Picchietti DL, Garcia-Borreguero D, Ondo WG, Walters AS, Winkelman JW, et al. Restless legs syndrome/Willis-Ekbom disease diagnostic criteria: updated International Restless Legs Syndrome Study Group (IRLSSG) consensus criteria--history, rationale, description, and significance. Sleep Med 2014;15:860-73.

2. Ohayon MM, O'Hara R, Vitiello MV. Epidemiology of restless legs syndrome: a synthesis of the literature. Sleep Med Rev 2012;16:283-95.

3. Earley CJ, Connor J, Garcia-Borreguero D, Jenner P, Winkelman J, Zee PC, et al. Altered brain iron homeostasis and dopaminergic function in Restless Legs Syndrome (Willis-Ekbom Disease). Sleep Med 2014;15: 1288-301.

4. O'Keeffe ST, Gavin K, Lavan JN. Iron status and restless legs syndrome in the elderly. Age Ageing 1994;23:200-3.

5. Sun ER, Chen CA, Ho G, Earley CJ, Allen RP. Iron and the restless legs syndrome. Sleep 1998;21:371-7.

6. Allen RP, Adler CH, Du W, Butcher A, Bregman DB, Earley CJ. Clinical efficacy and safety of IV ferric carboxymaltose (FCM) treatment of RLS: a multi-centred, placebo-controlled preliminary clinical trial. 
Sleep Med 2011;12:906-13.

7. Cho YW, Allen RP, Earley CJ. Clinical efficacy of ferric carboxymaltose treatment in patients with restless legs syndrome. Sleep Med 2016;25: 16-23.

8. Simpson IA, Ponnuru P, Klinger ME, Myers RL, Devraj K, Coe CL, et al. A novel model for brain iron uptake: introducing the concept of regulation. J Cereb Blood Flow Metab 2015;35:48-57.

9. Earley CJ, Connor JR, Beard JL, Malecki EA, Epstein DK, Allen RP. Abnormalities in CSF concentrations of ferritin and transferrin in restless legs syndrome. Neurology 2000;54:1698-700.

10. Connor JR, Boyer PJ, Menzies SL, Dellinger B, Allen RP, Ondo WG, et al. Neuropathological examination suggests impaired brain iron acquisition in restless legs syndrome. Neurology 2003;61:304-9.

11. Connor JR, Ponnuru P, Wang XS, Patton SM, Allen RP, Earley CJ. Profile of altered brain iron acquisition in restless legs syndrome. Brain 2011;134 (Pt 4):959-68.

12. Lopez A, Cacoub P, Macdougall IC, Peyrin-Biroulet L. Iron deficiency anaemia. Lancet 2016;387:907-16.

13. Allen RP, Picchietti D, Hening WA, Trenkwalder C, Walters AS, Montplaisi J, et al. Restless legs syndrome: diagnostic criteria, special considerations, and epidemiology. A report from the restless legs syndrome diagnosis and epidemiology workshop at the National Institutes of Health. Sleep Med 2003;4:101-19.

14. Winkelman JW, Armstrong M), Allen RP, Chaudhuri KR, Ondo W, Trenkwalder C, et al. Practice guideline summary: treatment of restless legs syndrome in adults: Report of the Guideline Development, Dissemination, and Implementation Subcommittee of the American Academy of Neurology. Neurology 2016;87:2585-93.

15. Li J, Moore H 4th, Lin L, Young T, Finn L, Peppard PE, et al. Association of low ferritin with PLM in the Wisconsin Sleep Cohort. Sleep Med 2015;16:1413-18.

16. Earley CJ, Connor JR, Beard JL, Clardy SL, Allen RP. Ferritin levels in the cerebrospinal fluid and restless legs syndrome: effects of different clinical phenotypes. Sleep 2005;28:1069-75.

17. Earley CJ, Barker PB, Horská A, Allen RP. MRI-determined regional brain iron concentrations in early- and late-onset restless legs syndrome. Sleep Med 2006;7:458-61.

18. Zacharski LR, Ornstein DL, Woloshin S, Schwartz LM. Association of age, sex, and race with body iron stores in adults: analysis of NHANES III data. Am Heart J 2000;140:98-104.

19. Allen RP, Bharmal M, Calloway M. Prevalence and disease burden of primary restless legs syndrome: results of a general population survey in the United States. Mov Disord 2011;26:114-20.

20. Allen RP, Picchietti DL, Auerbach M, Cho YW, Connor JR, Earley CJ, et al. Evidence-based and consensus clinical practice guidelines for the iron treatment of restless legs syndrome/Willis-Ekbom disease in adults and children: an IRLSSG task force report. Sleep Med 2018;41:27-44.

21. Trenkwalder C, Högl B, Benes H, Kohnen R. Augmentation in restless legs syndrome is associated with low ferritin. Sleep Med 2008;9:572-4. 